\title{
Protée
}

\section{Fonction rhétorique de la référence intermédiatique : sida, témoignage et intermédialité}

\section{Michel Fournier}

Volume 28, numéro 3, 2000

Mélancolie entre les arts

URI : https://id.erudit.org/iderudit/030606ar

DOI : https://doi.org/10.7202/030606ar

Aller au sommaire du numéro

\section{Éditeur(s)}

Département des arts et lettres - Université du Québec à Chicoutimi

ISSN

0300-3523 (imprimé)

1708-2307 (numérique)

Découvrir la revue

Citer cet article

Fournier, M. (2000). Fonction rhétorique de la référence intermédiatique : sida, témoignage et intermédialité. Protée, 28(3), 75-84.

https://doi.org/10.7202/030606ar
Résumé de l'article

Plusieurs manifestations culturelles liées au sida sont marquées par la présence d'une intertextualité faisant appel aux productions de différents médias. Ces références prennent part à la construction d'un objet sémiotique particulier et se présentent comme autant d'appels destinés à informer sa réception. Cet article se propose de mettre en relief la fonction rhétorique de la référence intermédiatique, en prenant le témoignage pour objet. En plus de participer à la constitution d'une figure d'énonciation destinée à donner au témoignage son efficace, ces références conduisent à la formation d'un espace commun, qui est lui-même traversé par une série de conflits sociaux. Ce texte cherche alors à montrer comment les références intermédiatiques inscrivent la prise de parole dans un nouvel espace symbolique, qui émerge à la croisée de différents médias. 


\section{CONCTION RHÉtorique DE LA RÉFÉREN CE INTERM ÉDIATIQ U E: SIDA, TÉMOIGNAGE ET INTERM ÉDIALITÉ \\ MICHEL FOURNIER}

C'est sans doute par la rumeur, ce plus vieux média du monde, pour reprendre l'expression de Jean-Noël Kapferer ${ }^{1}$, que le sida a commencé à prendre corps dans l'univers des discours ${ }^{2}$. Et c'est de ce bruit que le discours des médias semble avoir émergé, rendant alors distinct le murmure, pour s'en nourrir ou l'endiguer. Face à un discours qui, dans bien des cas, était empreint d'une tendance à la stigmatisation, la prise de parole s'est faite d'autant plus essentielle. Cette dernière s'est alors accompagnée de la recherche d'une scène, d'un espace qui serait apte à l'accueillir. Très tôt, les nouveaux médias ont été appelés à en tenir lieu ${ }^{3}$. Aux discours d'usage des différents médias s'est ajoutée une série de manifestations artistiques, qui ont déployé une véritable esthétique de la contamination; contamination des genres et des discours, mais aussi des médias ${ }^{4}$. Pensons aux recueils où le texte, poème ou témoignage, alterne avec la photographie; pensons aussi à ces romans qui se sont faits films, comme Les Nuits fauves de Cyril Collard. À travers d'autres manifestations, le discours relatif au sida a pris part au développement de nouveaux genres, qui tirent parti de possibilités technologiques relativement récentes; le journal-vidéo par exemple.

"Véritable laboratoire d'expérimentation» de pratiques tant éthiques qu'esthétiques ${ }^{5}$, les œuvres du sida ont mis en relief d'une façon exemplaire cette «intersection où se croisent les médias qui caractérisent la production culturelle contemporaine " ${ }^{6}$, dont rend compte l'intermédialité. Dans cet article, j'aborderai un aspect de cette interaction, qui prend peut-être place en périphérie de ce phénomène, mais qui fait aussi amplement écho à cette "croisée des médias». Si plusieurs manifestations culturelles témoignent matériellement de cette interaction en émergeant à la rencontre de différents médiums, d'autres s'inscrivent sur leurs frontières; par exemple, l'émission télévisée qui a le livre pour objet. Enfin, d'autres, celles qui m'intéresseront ici, mettent en jeu ce que l'on pourrait appeler la «référence» intermédiatique.

\section{DE LA RÉFÉRENCE INTERMÉDIATIQUE}

Les références «intermédiatiques» font appel, soit à un autre média, soit à des productions d'autres médias, en tant que leur provenance devient significative. Si 
le terme de «référence» implique qu'il n'y a pas de changement de médium, ces références ne sont cependant pas sans modifier la relation qu'entretient le lecteur ou le spectateur avec le média dans lequel elles apparaissent. Les recherches sur les pratiques de lecture offrent déjà une réflexion sur ces enjeux, en soulignant la façon dont la lecture se transforme en fonction des médias qui l'entourent. L'intermédialité peut alors s'entendre à la manière de l'intertextualité qui, en plus d'obliger une sortie de la clôture du texte, nous engage à aborder le rapport au référent comme étant lui-même traversé par les médiations d'un ensemble de discours ${ }^{7}$. Je qualifie ces références d'intermédiatiques pour deux raisons. Premièrement, la distinction terminologique d'avec l'intertextualité vise ici à mettre l'accent sur des médiations que l'extension du terme "texte», par moment, risque d'occulter. Deuxièmement, le terme «intermédiatique» nous permet de conserver l' «entre» du préfixe «inter» et, par là, d'insister sur le fait que ces références, dans bien des cas, semblent aménager, ou du moins esquisser, de véritables passages entre ces médias.

À demeurer dans les limites du texte, on peut envisager ces références comme n'étant destinées qu'à produire, dans un univers fictif, une représentation des médias, au même titre que peuvent être représentés d'autres phénomènes ou d'autres objets. Par contre, lorsqu'on aborde le texte comme objet esthétique ou comme objet culturel, qu'on tient compte des pratiques dans lesquelles il prend place et des conditions de sa réception, ces références apparaissent comme autant d'appels destinés à informer le rapport que le lecteur entretient avec le texte et à constituer une saisie particulière de ce dernier. Ainsi, un texte, qui se présente comme une suite de photographies ou qui met en scène un narrateur écrivant une caméra à la main, semble chercher un effet de lecture différent de celui qui se contente d'«il était une fois». Les références intermédiatiques prennent donc part à la construction d'un objet sémiotique particulier, qui en appelle à son tour de diverses pratiques symboliques, et diffère en partie des autres objets convoquant le même support ou prenant place dans la même institution.

Plusieurs œuvres traitant du sida sont habitées par ce qui, à première vue, peut apparaître comme une véritable pulsion à citer, à nommer d'autre ouvres qui proviennent de différents médias. Ces références peuvent alors viser à greffer le phénomène dans la culture, en opérant une contamination du symbolique. En fait, c'est tout un imaginaire qui doit se reconstruire autour de la maladie. Cet imaginaire, dans bien des cas, a été celui de l'exclusion. Le discours social a tout d'abord fait appel au fond archaïque du bouc émissaire, avant d'associer le sida à la nouvelle culture des réseaux; du «sida mental» des jeunes aux virus de toutes sortes ${ }^{8}$. C'est à partir de ce nouvel imaginaire qu'une série de renversements semble s'être opérée. Cette nouvelle culture a en quelque sorte offert l'espace nécessaire pour réaliser une véritable greffe symbolique et introduire ce nouveau phénomène dans le territoire culturel.

Dans les pages qui suivent, je limiterai mon propos à la fonction rhétorique de la référence intermédiatique, en prenant le témoignage pour objet. Ce genre, qui occupe une place importante dans le corpus du sida, semble en appeler de l'intermédialité dans sa définition même, puisqu'il se définit souvent davantage en fonction des productions d'autres médias (des reportages de la presse écrite à ceux de la télévision) qu'en relation avec les autres genres littéraires. La pratique du témoignage participe également à la remise en question des frontières entre espaces public et privé, qui fait suite aux transformations de l'espace médiatique. De plus, par sa revendication d'une certaine forme d'immédiateté, le témoignage peut s'avérer symptomatique de la "vidéosphère " 9 dans laquelle nous évoluons. Enfin, le témoignage se présente comme un discours d'usage ${ }^{10}$ et vise dans la plupart des cas une finalité pratique: présenter ou dénoncer une situation en vue de sa transformation. Il nous fait ainsi passer des pratiques sémiotiques aux pratiques culturelles en général. 


\section{SITUATION DU SPECTATEUR}

Cette réflexion sur l'intermédialité a pour point de départ une scène rencontrée dans le témoignage d'une jeune personne vivant avec le sida, Barbara Samson. L'auteure de ce témoignage a été contaminée, lorsqu'elle était mineure, par un homme qui se savait séropositif. La situation se fait d'autant plus dramatique que cette contamination a lieu dans un centre médical, où elle est envoyée à la suite d'une tentative de suicide. De plus, les autorités du centre étaient au courant de la séropositivité de cet homme. Ce témoignage cherche alors à remettre en question la valeur du secret professionnel, en inscrivant cet enjeu dans une problématique de la diffusion de l'information en général ${ }^{11}$. Dans un chapitre intitulé "Cinémas", la narratrice assiste à une projection des Nuits fauves de Cyril Collard. L'écran lui offre une représentation de sa propre histoire et la narratrice met en relief les analogies entre ce film et sa vie:

Reste l'histoire principale des Nuits fauves, l'amour entre Laura et Jean. L'amour entre Barbara et Antony.

Elle a dix-sept ans, elle est amoureuse. Il est séropo, il le sait, et il ne lui dit pas tout de suite. Ça me ressemble énormément. Et si je pleure, c'est par réminiscence. Parce que je revois trop les moments clefs de ma courte vie. ${ }^{12}$

Le chapitre se transforme en un commentaire du film, et surtout de sa réception:

J'y vais toute seule. Puisque tout le monde dit: Collard, c'est génial. Collard à la télévision, Collard dans les magazines, Collard partout. Le sida devient star [...] Collard est un mythe créé par les médias. Celui dont on dit qu'il est le premier à parler du sida et des jeunes, sans faux-fuyant ni retenue. (p. 175)

Et plus loin:

Je ne sais pas pourquoi on crie au génie fauve. J'en pleure, seule dans le noir. Je sors du cinéma les yeux gonflés, ébranlée, détruite. Tout cela manque de mesure. Les médias exagèrent; fascinés par cette saloperie de maladie, ils en font un show. (p. 179)

Les références intermédiatiques donnent ainsi lieu à une pratique métatextuelle qui a pour objet non seulement le film de Collard, mais aussi la couverture médiatique qui en est faite. Ces références sont loin d'être gratuites, puisque tout le témoignage de Samson est porteur d'une rhétorique destinée à assimiler l'homme qui l'a contaminée au héros des Nuits Fauves, et à contester à Collard le titre de représentant, de "porte-parole» d'une génération.

Au film de Collard, la narratrice opposera premièrement la vie réelle, son histoire vécue du point de vue de la victime. Quelques pages plus loin, un chapitre s'intitule «Réalités». À la fin du film, l'héroïne peut s'en tirer saine et sauve, puisque ce n'est que de la fiction. Bien que les Nuits fauves s'inscrivent dans ce courant qui va du livre au film et à la télé «vérité», le média cinématographique (tel qu'il est alors représenté) se pose comme une entrave à l'effet de véracité visé. "Ce n'est que du cinéma», répète Samson, et le cinéma ici inclut tout le dispositif médiatique qui l'entoure. Dans le texte de Samson, le récit de la victime s'oppose non seulement à celui du bourreau, mais aussi à celui de la star, au pouvoir du grand écran. Cet enjeu se fait encore plus manifeste lorsque Samson oppose à Collard une autre figure d'artiste sidéen, figure littéraire cette fois:

Hervé Guibert a fait des livres magnifiques sur le sujet. Mais on ne l'a pas pour autant élevé au titre de porte-parole d'une génération. Il était trop prudent, trop grave, trop conscient.

(p. 178-179)

Cette opposition entre Collard et Guibert n'est pas exclusive au texte de Samson; on la retrouve dans la critique, mais aussi dans d'autres témoignages ${ }^{13}$. À travers cette opposition, ce n'est pas seulement deux œuvres qui seront mises en concurrence, mais deux espaces médiatiques: le cinéma et la littérature. Si les références littéraires se multiplient dans ce texte, le cinéma ne réapparaît que pour être associé à la zone que Samson explore et condamne, monde du délit et de la drogue dans lequel évolue l'homme qui l'a contaminée et qui est le monde du «déjà vu au cinéma».

Au vécu que Samson oppose à l'espace cinématographique s'ajoutera un autre média: le petit 
écran. La télévision apparaît dans ce texte à travers le récit que fait la narratrice de son passage à la soirée «Sidaction». Ce média est alors d'autant plus investi des valeurs de véracité que l'émission en question, une soirée-bénéfice pour la lutte contre le sida, est à la fois exemplaire du direct et de la télé-vérité. En fait, ce texte qui culmine avec le passage de la narratrice à l'écran obéit tout au long à l'impératif voulant qu'il doit y avoir image, en nous racontant la genèse de cette dernière. Son écriture, visant à produire cet oubli du texte qui prend part à la stratégie du vécu, semble par moments chercher à donner au lecteur l'impression d'être devant un écran. À cette fin, tout un lexique sera mobilisé; par exemple, dans des phrases comme celle-ci: "Je l'imagine très bien; gros plan sur lui : il va rencontrer une autre fille» (p.236, je souligne).

Dans le processus de représentation que met en jeu le témoignage de Samson, les médias sont investis de diverses valeurs qui relèvent non seulement de l'économie des discours ${ }^{14}$, mais aussi de la configuration que le texte construit. $\mathrm{Si}$, dans le texte de Samson, le cinéma est associé au monde de l'illusion (que «soulignent» la facticité de l'univers des stars et son écho médiatique), la littérature a pour elle le sérieux, voire la "gravité». La télévision, pour sa part, est associée à la véracité (en opposition au cinéma) et surtout à la "représentativité», puisqu'elle offre une scène qui prend place, comme le met en relief le titre même du chapitre ( Devant la France entière») où le passage de Samson est relaté. Samson définit alors sa propre position d'énonciation en fonction de ces différents médias, et le texte nous présente un parcours marqué par l'acquisition d'un pouvoir symbolique destiné à donner au témoignage son efficace ${ }^{15}$.

\section{ETHOS FIN DE SIĖCLE}

Peu après le récit de l'apparition de la narratrice à la soirée "Sidaction", le texte de Samson se conclut par ces mots, qu'accompagne une citation de Musset:

J'ai rencontré un homme "négatif", je suis une jeune femme "positive». Il le sait, je le sais; nous savons. Puisque je suis un peu le symbole de la Confession d'un enfant du siècle. Alors s'assit sur un monde en ruines/une jeunesse soucieuse. (p. 239)

Au «lieu» d'énonciation, que dessinait la configuration mise en place par les références à différents médias, s'ajoute alors une figure qui se développe tout au long du texte et dont la fonction n'est pas sans rappeler celle de l'ethos en rhétorique.

L'ethos ou caractère de l'orateur, comme le rappelle Gilles

Declercq, désigne l'image morale de l'orateur et l'effet de cette image sur l'auditoire, dont la bonne ou manvaise disposition détermine le succès ou l'insuccès du discours. 16

L'expression «jeunesse soucieuse» traduit cet ethos que le texte de Samson tentera de construire par un ensemble de références culturelles, et qui s'oppose à la jeunesse insouciante que représente la "génération Collard".

Pour se faire le nouveau porte-parole d'une génération, la narratrice doit pouvoir incarner cette jeunesse, et c'est ici qu'intervient une esthétique qu'elle partage avec Collard. À certains moments, le témoignage de Samson apparaît comme une véritable reprise de la version filmique des Nuits fauves. Si le film de Collard s'ouvre sur une scène où Jean (le personnage principal), du haut de quelques étages, regarde Laura qui descend d'une motocyclette, le texte de Samson débute par une scène où la narratrice, descendant de voiture, se voit regardée par Antony (l'homme qui l'a contaminée) du haut d'un balcon. L'incipit, qui se présente comme une «réponse» aux Nuits fauves, inscrit alors un renversement de perspective qui se traduit dans les jeux de focalisation: vision de l'homme qui regarde (en plongée) / vision de la femme regardée (en "contre-plongée»). Cette jeunesse «soucieuse» doit cependant se distinguer de la génération insouciante que Collard représente. C'est ici que prennent place les références «littéraires»: Musset, Céline, Rimbaud surtout. Les références littéraires ne sont pas absentes du film et du roman de Collard, mais elles prennent une valeur différente dans le texte de Samson, qui semble chercher à inscrire une dimension «littéraire» 
renvoyant moins à une littérarité qu'à une valeur «littérature», ici synonyme de "sérieux», de "conscience» et de "gravité».

Ces références inscrivent également une démarcation entre une culture nationale, celle de la narratrice, et la culture de l'homme qui l'a contaminée. Cette opposition est implicitement présente dans ce texte qui, de façon allusive, souligne l'origine "étrangère» de cet homme, à travers une scène où une transaction de drogue s'effectue en langue arabe. Des références musicales prennent place dans cette opposition, avant de souligner à leur tour la distinction entre deux générations. Ainsi, lorsque Antony évoque Jim Morrison, la narratrice précise qu'il s'agit d'une «autre époque pour [elle]» (p.28). Plus loin, le conflit des "générations» sera explicitement évoqué, et Antony sera présenté comme faisant partie de la même génération que Collard ${ }^{17}$. Enfin, une autre distinction apparaît, celle des classes sociales. À de nombreuses reprises, la narratrice souligne cette distinction qui se traduit sur le plan du lexique utilisé. Si la narratrice utilise un vocabulaire connotant la «jeunesse» qu'elle représente, le registre dans lequel il se situe se distingue de celui du discours d'Antony, qui renvoie au discours "populaire» de la zone $^{18}$.

Les références intermédiatiques apparaissent tout au long du récit, qui est lui-même ponctué par le motif de la télévision. Ainsi, la vie de la narratrice est vue à travers une série de photos de famille, et la «fracture» dans son propre récit correspond au moment où elle cesse d'apparaître dans l'album familial (p.223). De même, le jeu de la séduction entre la narratrice et Antony prend forme autour d'un échange de références musicales (Morrison/ Gainsbourg) et, surtout, d'un cahier de poèmes. Au premier crime de cet homme s'en ajoute un second, qui conduit cette fois à la rupture. Cette dernière s'effectue au moment où la narratrice découvre que les poèmes qu'il prétendait écrire pour elle ne sont que des copies des textes de Morrison et de Rimbaud ${ }^{19}$. Certes, ces «références» relèvent de l'histoire racontée, qui est elle-même une histoire vécue. Mais si l'histoire qu'il relate doit être tenue pour véridique, ce témoignage (écrit en collaboration) est l'objet d'une construction élaborée; ne serait-ce que sur le plan narratif, à travers un récit qui, après un début in medias res, est fait de passages rétrospectifs et de digressions.

De plus, si certaines références s'inscrivent dans l'histoire, d'autres relèvent de la narration. Par exemple, le chapitre où s'effectue la rupture définitive entre la narratrice et Antony débute par la scène suivante: la narratrice est dans un lit d'hôpital et écoute Gainsbourg à travers les écouteurs de son "walkman». Le texte enchaîne avec un extrait d'une chanson de Gainsbourg, nous laissant croire qu'il s'agit de la chanson écoutée: «Amour, hélas, ne prend qu'un «m», faute de frappe, c'est haine, pour aime..." (p.213). La voix de Gainsbourg était déjà apparue au moment où la narratrice attend les résultats du test de dépistage :

Gainsbourg chante:

Le compte avait commencé à rebours,

était-ce vertige, déveine, qui sait.

Un voyage, un seul aller au long cours

d'où l'on ne revient jamais.

Sorry Angel... (p. 149)

Contrairement au premier exemple, où le texte pouvait «reproduire» ce que jouait le walkman, aucun élément de la diégèse (walkman, radio, télévision) n'apparaît ici comme la "source» de cette chanson, qui n'est pas sans évoquer la trame sonore d'un film. Les paroles de Gainsbourg mettent en relief les moments clefs de l'histoire: le renversement amour/haine a lieu quand joue "Haine pour aime», et le début de la «chute» de la narratrice a pour musique de fond «Sorry Angel». En plus de mettre en relief des éléments importants du récit, ces paroles s'opposent à celles de Morrison, qui sont elles aussi disséminées tout au long du texte, et partagent certains motifs (comme celui de l'ange) avec les chansons de Gainsbourg. Les événements de l'histoire trouve ainsi un écho dans le discours et l'opposition Samson/Antony-Collard se transforme en duel Gainsbourg/Morrison. 
Les références à Rimbaud s'inscrivent dans une constellation encore plus manifeste ${ }^{20}$. Lorsque la narratrice découvre la supercherie dont elle est victime, c'est principalement à travers des textes de Morrison et un poème de Rimbaud intitulé «Roman " (p.206-207). Un passage de ce poème, cité à deux reprises lors de la scène, constitue le titre de ce témoignage ( on est pas sérieux quand on a dix-sept ans»), qui est aussi celui de son premier chapitre. Les deuxième, troisième et dixième chapitres sont à leur tour intitulés à partir d'extraits de ce poème. Si le poème se fragmente et se dissémine jusqu'à couvrir le paratexte, il s'inscrit également dans le corps du texte, sans pour autant que sa présence soit motivée par les événements de l'histoire (la lecture d'un texte, par exemple). Un long extrait prend place après la première relation sexuelle de la narratrice, et un autre passage s'inscrit au moment où elle rejoint Antony à Aix (p. 72). Une dernière référence à Rimbaud apparaît dans le dixième chapitre, dont le titre («Des parfums de bière») fait référence, cette fois, à des scènes où l'alcoolisme se mélange à la toxicomanie:

Rimbaud n'est plus. Le roman de mes dix-sept ans a le parfum amer d'un mélo de zonarde. Poésie, rêve, mon refuge meurt sur un quai de gare. Je n'irai plus jamais sous les tilleuls de la promenade. (p.128)

Les références à ce texte de Rimbaud soulignent son statut de "poème», à la manière dont les références aux Nuits fauves en appelaient du "cinéma".

De même que les références intermédiatiques prenant place au niveau de l'histoire produisent un effet de réel qui se traduit en effet de véracité, les références qui s'inscrivent au niveau de la narration semblent générer un effet de présence visant, cette fois, à créer l'illusion d'une "communication", illusion recherchée par le témoignage. Si l'intertextualité marque l'appartenance d'un texte à une tradition et le situe à l'intérieur de cette dernière, la référence intermédiatique produit, dans ce témoignage du moins, un effet d'extériorité; comme si le texte prenait place dans un interstice entre les médias et acquérait par là une valeur de "réalité». Les paroles qui font suite au récit du passage de la narratrice à l'écran cherchent à leur tour à créer une illusion d'immédiateté. Le ton adopté est celui de la confidence et en appelle du registre de l'oralité: "Voilà, j'ai fini. “Je suis venue vous dire que je m'en vais”. Je vais survivre. Travailler. Me bagarrer pour l'information» (p.239).

\section{DES OBJETS MÉDIATIQUES}

Les objets convoqués par Samson (film de Collard, livres de Guibert) sont eux-mêmes déjà pris en charge par un ensemble de discours, auxquels ce témoignage fait écho. Depuis le début de la pandémie, la construction sociale de la maladie a été intimement liée à son traitement par les médias. Comme le souligne Alain Emmanuel Dreuilhe dans Corps à corps. Journal de sida: «Progrès de la technique, le SIDA est la première épidémie en direct, tout comme la guerre du Viêt Nam était la première guerre télévisée "21. Si plusieurs manifestations artistiques ont opéré une critique du discours médiatique, les médias se sont à leur tour emparés des figures d'artistes sidéens. Collard et Guibert sont, en France, parmi les plus importantes. Par l'esthétique qu'il met en jeu, le film de Collard 22 inscrit le sida dans une culture qui se veut celle de la fin du millénaire. Image vidéo, monde du clip, rythme rapide, scène de course en voiture et dialogues brisés par la médiation du répondeur font alors écho à l'univers de la drogue et à une sexualité marginale, allant des rencontres sous les ponts aux séances sadomasochistes. Il est somme toute peu risqué d'affirmer que l'intermédialité mise en scène par ce film est pour beaucoup dans le succès de ce dernier. Prenons pour exemple l'article de Frédéric Strauss, paru dans Les Cahiers du Cinéma:

La communication est affaire de synchronisme et Les Nuits

fauves est le premier film qui soit synchrone avec la

communication actuelle: ce n'est plus seulement un duel individuel, la recherche d'un franc face à face intime, mais l'élaboration (douloureuse et dynamique) d'un dialogue traversé par les bruits du monde, par les interférences d'une explosion des modes d'expression. Roman, clips, films, musique, Cyril Collard a restitué son expérience de toutes ces formes d'expression dans 
un cinéma libéré de sa relation étroite, incestueuse et intimidante à la seule cinéphilie. 23

En fait, ce qui se dégage de la réception de ce film autour duquel les médias ont entretenu l'illusion autobiographique, c'est l'idée d'une nouvelle culture dont il serait l'emblème, culture qui serait celle d'une "génération sida".

Hervé Guibert, de son côté, est l'auteur d'une œuvre assez vaste et devient une véritable figure médiatique en passant à l'émission télévisée Apostrophe, avec son roman À l'ami qui ne m'a pas sauvé la vie ${ }^{24}$. Le texte prend alors place dans un dispositif qui use des ressources du médium télévisuel pour l'inscrire sous le régime de la référence et imposer une lecture plus ou moins autobiographique ${ }^{25}$. Ici, il est intéressant de noter la façon dont les catégories génériques s'estompent pour laisser place à des objets qui sont les produits d'une intermédialité. L'appel à une lecture référentielle semble parfois moins provenir des caractéristiques propres au texte que de sa transformation en objet médiatique. Catégories poétiques et catégories médiatiques diffèrent et, en ce qui concerne le roman de Guibert, la prise en charge médiatique nous fait passer de l'autofiction à l'autobiographie. À travers sa réception, À l'ami qui ne m'a pas sauvé la vie se métamorphose également en texte à clefs. Sous le couvert d'un personnage nommé Musil, Guibert révélerait des détails de la vie intime de Michel Foucault. Le texte engage alors un débat sur le respect de la vie privée. Ce débat, dans son traitement médiatique, ne se limitera pas au texte de Guibert, mais prendra à partie l'ensemble de l'institution littéraire et le statut du texte qui s'y inscrit. Les titres de certains articles, comme celui de Jérôme Garcin, «La littérature a-t-elle tous les droits?», en sont emblématiques ${ }^{26}$. Tout comme Samson faisait des Nuits fauves du "cinéma», Garcin s'attaque à la «littérature» lorsqu'il dénonce le texte de Guibert.

Ainsi, en se référant au film de Collard et aux romans de Guibert, Samson fait appel non seulement à des œuvres, mais à des objets médiatiques complexes qui se construisent à travers leur réception et sont alors investis d'une charge axiologique. Par ces références, Samson prend place sur une scène virtuelle qui émerge comme à la croisée des médias, et sur laquelle évoluent ces figures. Ce n'est plus à l'inscription culturelle de la maladie que participent les références intermédiatiques, mais à la constitution d'un espace symbolique où prennent place d'autres enjeux. La citation, en traversant les frontières, en appelle moins d'une tradition que de cet espace. À travers les conflits sociaux dont ces figures sont investies - négligence quant à la transmission ( Collard»), profanation de la vie privée («Guibert»)-, la scène se fait littéralement tribunal et le témoignage prend son sens juridique. Ce témoignage prend alors part au phénomène de médiatisation du juridique, et la dénonciation d'un acte criminel, la transmission volontaire, cède ici place à la condamnation d'une figure médiatique ${ }^{27}$. Dans ce texte, qui se fait le lieu de pratiques allant de la citation au commentaire, s'opère une redéfinition de l'autorité qui résulte non seulement de l'expérience dont le témoignage rend compte, mais aussi de la capacité de la narratrice à incarner les valeurs dont différents médias peuvent être investis.

\section{PRISE DE PAROLE ET ESPACE SYMBOLIQUE}

Ces références intermédiatiques ne font pas que prendre part à la signification du témoignage, elles participent également de l'efficace à laquelle il prétend. Plus loin dans ce texte, Samson inscrit un plaidoyer sous la tutelle de Guibert, en se référant au titre de son roman, À l'ami qui ne m'a pas sauvé la vie: "À ce médecin qui ne m'a pas sauvé la vie, je voudrais dire: "Si vous en voyez un autre, ou une autre, rompez le secret professionnel; faites-le, pour qu'il ou elle vive" " (p. 228). Le "cas (médiatique) Guibert» fait ici fonction de précédent en matière de "bris» du secret relatif à la vie privée. À travers cet appel au discours médiatique, le texte de Samson nous entraine de la sphère juridique à celle de l'opinion, passage lié à un désinvestissement du pouvoir officiel, qui est luimême mis en scène dans ce témoignage et qui n'est pas sans être empreint des échos du scandale du sang 
contaminé28. Enfin, en faisant appel à ces figures médiatiques (Guibert, Collard), le texte construit une médiation entre le public et une réalité qu'il pourrait avoir tendance à rejeter; il prend ainsi part à la formation d'un «lieu», d'un espace commun ${ }^{29}$.

En plus de participer à la formation de cet espace et de construire une figure d'énonciation destinée à donner au témoignage son efficace, les références cinématographiques et télévisuelles qui ponctuent le texte de Samson mettent en relief le pouvoir de l'image dans la culture actuelle. Il doit y avoir image, le texte de Samson semble obéir à cet impératif ${ }^{30}$. Si l'image se veut garante du fait que quelque chose a existé, le texte de Samson aura pour leitmotiv: l'image a eu lieu ${ }^{31}$. Et si le texte y répond en représentant le passage de la narratrice à l'écran, il a lui-même pour origine une déficience de l'image. En interrompant le récit de ce passage à l'écran, la narratrice imagine le moment où l'homme qui l'a contaminée décidera d'en séduire une autre, pour la contaminer à son tour. Cette idée donne lieu à un constat d'impuissance. Le seule usage adéquat de l'image, qui consisterait à exhiber la photo de cet homme, est interdit par la loi:

Que faire, je ne peux pas aller devant cette caméra et dire: «il

s'appelle Antony Machin, il a trente ans, regardez bien sa photo, ne l'aimez pas!» Je n'ai pas le droit. Je suis démunie. Je n'ai que ce témoignage... (p. 236)

L'image interdite engendre le récit et donne ainsi lieu à l'image racontée ${ }^{32}$.

Enfin, les références intermédiatiques semblent déplacer ce témoignage des marges du littéraire pour l'inscrire dans un espace qui, cette fois, émerge à la croisée des médias. Ainsi, la référence intermédiatique ne fait pas que participer d'un effet de réel ou de la mise en scène d'un quotidien traversé par le discours des médias; elle engage la production d'un espace symbolique ${ }^{33}$ où une série de pratiques se déploie. C'est dans ce même espace, où les frontières entre cultures populaire et savante s'estompent, que des manifestations artistiques seront appelées à prendre place, à travers leur réception. L'esthétique de la contamination que partagent œuvres d'arts et témoignages semblent alors conduire à la genèse d'un nouvel espace où, déjà, s'affrontent différents discours. À travers la référence intermédiatique, les manifestations culturelles liées au sida en appellent de cet espace et prennent part à sa construction. Cette scène, elle-même traversée par un ensemble de conflits, se fait à son tour le lieu de l'inscription culturelle de la maladie et d'une prise de parole qui lui est liée.

\section{N O TES}

1. J.-N. Kapferer, 1987.

2. Cet article fait suite à des travaux menés dans le cadre d'un groupe de recherche sur l'identité sexuée dans les littératures du sida dirigé par M. Delvaux à l'Université du Québec à Montréal.

3. On n'a qu'à penser à la façon dont l'association ACT UP a rapidement su tirer parti des ressources d'Internet pour diffuser diverses informations. D'autres pratiques ont fait un nouvel usage de «médias" plus traditionnels qui ont trouvé un prolongement électronique. Par exemple, le projet de la courtepointe des noms ("NAMES Project AIDS Quilt") a mis en place une subversion de certaines pratiques commémoratives traditionnelles, et se poursuit dans un site Internet. Sur le "NAMES Project AIDS Quilt», voir P.S. Hawkins, 1993: 752-779.

4. L'œuvre de H. Guibert en est un bon exemple, où photographie, littérature et vidéo se répondent. Sur l'œuvre de Guibert, voir le numéro spécial que La Revue des Lettres Modernes lui a consacré ("au jour le siècle 2", 1997), ainsi que celui de Nottingham French Studies (vol. 34, nº 1, printemps 1995).

5. Voir G. Bibeau et R. Murbach, 1991: 5-11.

6. Je reprends ici la définition donnée dans le texte de présentation du colloque du Centre de recherche sur l'intermédialité (CRI).

7. En ce qui concerne la diffusion de cette notion, voir M. Angenot, 1983: 121-135.

8. Voir I. Rieusset-Lemarié, 1992.

9. Voir R. Debray, 1993.

10. Sur la distinction discours fictif/discours d'usage, voir G. Thérien, 1989:139-157.

11. Le témoignage de Samson pose une série de problèmes sur le plan éthique, car s'il inscrit les enjeux d'une prise de parole qui se fait à partir d'une position marginalisée (celle d'une personne vivant avec le sida) et souligne le problème social et légal que représente la négligence quant à la transmission du virus, ce texte, à travers ses revendications, 
rejoint en partie certains discours qui, cette fois, tendent à la stigmatisation de certains groupes sociaux. En effet, après avoir insisté sur sa solidarité ("adolescente») avec la «marge », Samson opère un renversement (qui se traduit par un retour au centre que représente la famille) et s'attaque à ce qui deviendra la "zone", monde qui apparaît tout d'abord comme étant celui de la toxicomanie, mais dont le contenu est par moments «incertain". À travers la figure de l'homme qui l'a contaminée, le texte de Samson met en place une catégorie (la "marge ") à laquelle il finit par s'attaquer.

12. B. Samson, On est pas sérieux quand on a dix-sept ans, Paris, Fixot, 1994. Les prochaines références à ce texte se feront dans le corps du texte, entre parenthèses (p. 176).

13. Voir Le Couloir: une infirmière au pays du sida de F. Baranne qui, cette fois, adopte une perspective favorable à Collard aux dépens de Guibert.

14. Économie dans laquelle les médias finissent par être définis en fonction des autres médias qui les entourent; par exemple, le livre sera jugé traditionnel face à l' "avant-gardisme» des "nouveaux médias". 15. La critique des Nuits fauves prend part à la constitution d'un discours de vérité, a contrario, à travers la dénonciation de l'illusion que représente ce film (auquel la narratrice oppose sa propre expérience). Le passage de la narratrice à la télévision marque sa "reconnaissance" et en fait un nouveau porte-parole. Enfin, le témoignage lui-même est le lieu d'un passage à l'écriture, où les références littéraires donnent au discours son "sérieux", en plus de l'inscrire dans l'ordre d'une culture nationale.

16. G. Declercq, 1993: 47. Sur la relation entre ethos et énonciation, voir O. Ducrot, 1984 : 171-233.

17. "Collard, ce génie des médias, est de la même génération. Ils ont tous deux vécu sans courage, sans respect, ne pensant qu'à leur liberté, leur plaisir, leur désir» (p. 180).

18. Et qui se distingue de la "classe moyenne» à laquelle la narratrice appartient.

19. Cette révélation du plagiat est préparée longtemps à l'avance, même pour un lecteur qui ne connaît pas la provenance des extraits cités, dans les remarques que fait la narratrice à propos de la piètre orthographe de cet homme (qui détonne alors avec le contenu de ces textes). La distinction de classe réapparaît, puisque lorsque cet homme cherche à s'approprier une culture (bourgeoise) qui n'est pas la sienne, c'est à travers la modalité du délit; délit "scolaire" par excellence, le plagiat.

20. Céline fait l'objet d'un procédé similaire. La citation de cet auteur qui figure en épigraphe est reprise dans le corps du texte: "porteuse du plus grand malheur possible, comme dit Céline, le génie noir : “C'est peut-être cela que l'on cherche, rien que cela, le plus grand malheur possible, pour devenir soi-même avant de mourir" "(p. 179). Enfin, un extrait de cette citation trouve écho dans le titre de l'avant-dernier chapitre: "Le plus grand chagrin possible».

21. A. E. Dreuilhe, $1987: 154-155$

22. Les Nuits fauves de Collard sont un exemple intéressant de pratique intermédiatique et de la réception qui s'y inscrit. Il s'agit tout d'abord d'un roman, paru en 1989 chez Flammarion. Ce roman, qui ne connaît qu'un succès relatif, donnera lieu au film du même titre. Le film, cette fois, est une réussite, et le roman sera réédité en format de poche, sous couvert de l'affiche du film. Au succès commercial s'ajoute la reconnaissance institutionnelle et Les Nuits fauves se verront décerner le César du meilleur film en 1993.

23. F. Strauss, 1993: 6 .
24. Ce type d'émission constitue un bon exemple de manifestation, prenant place à la frontière d'un média et participant à son tour de ce dernier, ne serait-ce qu'en informant sa réception. Sur le passage de Guibert à Apostrophe, voir J.-P. Boulé, 1995: 112-120.

25. Comme le remarque Philippe Lejeune, la construction de l'objet, qui s'effectue premièrement dans le discours de l'animateur, s'appuie également sur les possibilités technologiques du média télévisuel. Par exemple, lorsque l'ouvrage dont il est question occupe l'écran, une fenêtre s'ouvre pour laisser place à l'image de l'auteur, qui apparaît comme au cour de son propre livre. Voir P. Lejeune, 1986: 87-99. Dans le cas du témoignage de Samson, c'est le paratexte qui occupe cette fonction. Le livre de Samson en effet use de diverses ressources visuelles. La couverture nous offre, en plus du titre qui se répète sur la frange et à l'endos du texte, une photo de l'auteure (à droite sous le titre et le nom de l'auteure). Le reste de la page est occupé par un extrait (qui ne figure pas exactement sous cette forme dans le texte) inscrit entre guillemets. Tout comme le titre, les dernières phrases de cet extrait sont écrites en rouge: "J'avais dix-sept ans. J'ai cru à une déclaration d'amour. C'était une phrase de mort. Elle m'est restée plantée dans le cœur». Le reste du texte est en noir, sur fond blanc. L'endos du livre reprend le même cadre, sans la photo de l'auteure, et offre un texte anonyme qui débute en faisant référence à la soirée «Sidaction": «le 7 avril 1994, Barbara Samson bouleversait des millions de téléspectateurs". Dans le texte qui suit, trois extraits sont écrits en rouge et sont d'autant plus en relief qu'ils ne suivent pas complètement le découpage syntaxique ( Contaminée par le virus du sida à dix-sept ans, lors de sa première histoire d'amour "; "Barbara a décidé de se battre"; "pour que l'amour ne soit plus jamais porteur de mort»). Les trois extraits, séparés à chaque fois par quelques lignes, donnent finalement un nouvel énoncé, qui prend la forme d'une formule choc. Le rouge fait écho à une symbolique du sang, qui renvoie alors non seulement à la question du sida mais aussi à la symbolique que met explicitement en œuvre le texte, et qui oppose la virginité de la narratrice au sang de sa première relation.

26. Garcin pose alors le problème en ces termes: «Il y a une mort intime comme il y a une vie intime, que la loi est censée protéger contre toute forme d'infraction. Mais la littérature abhorre les règlements et vomit les édits. Elle s'octroie des privautés éthiques au même titre qu'elle s'arroge des licences esthétiques, bénéficiant, par essence, d'une immunité tacite dont elle entend bien user chaque fois que la justice lui demande des comptes " (1990: 80).

27. Si l'homme qui a contaminé la narratrice est à plusieurs reprises traité de "criminel», il ne sera jamais question de la possibilité d'une démarche judiciaire dans ce texte.

28. Voir P. Champagne et D. Marchetti, 1994 : 40-62.

29. Sur la façon dont certains textes traitant du sida engagent un processus de décontamination qui conduit à la formation de cet espace commun, voir M. Delvaux, 1997 : 83-93.

30. En se faisant récit de l'image, le texte écrit lui confère une valeur supplémentaire, plutôt que d'être mis en opposition à cette dernière. 31. Mais le texte de Samson précise que cette «image» doit être lue selon les modalités de l'image "télévisuelle» et non pas "cinématographique».

32. Le récit conduira à nouveau à l'image, à travers la transformation de ce témoignage en série télévisée qui fera suite à sa réédition en format de poche.

33. J'emprunte cette notion à H. Merlin, 1994: 131-133. 


\section{RÉFÉREN CES BIBLIO G RAPH IQ U ES}

ANGENOT, M. [1983]: " L'intertextualité": enquête sur l'émergence et la diffusion d'un champ notionnel", Revue des sciences humaines, tome LX, no 189 , janvier-mars, 121-135.

BARANNE, F. [1994] : Le Couloir: une infirmière au pays du sida, Paris, Gallimard.

BibeAu, G. et R. Murbach [1991] : «Déconstruire l'univers du sida », Anthropologie et Sociétés, vol. 15, no 2-3, 5-11.

BOULÉ, J.-P. [1995] : «Hervé Guibert à la télévision : vérité et séduction ", Nottingham French Studies, vol. 34, n 1 , printemps, 112 120.

Champagne P. et D. Marchetti [1994] : «L'information médicale sous contrainte. À propos du scandale du sang contaminé ", Actes de la recherche en sciences sociales, $\mathrm{n}^{\circ}$ 101-102, mars, 40-62.

COllard, C. [1989]: Les Nuits fauves, Paris, Flammarion.

DEBRAY, R. [1993]: L'État séducteur: les révolutions médiologiques du pouvoir, Paris, Gallimard.

DECLERCQ, G. [1993] : L'Art d'argumenter. Structures rhétoriques et littéraires, Paris, Éd. Universitaires.

DELVAUX, M. [1997]: "Des corps et des frontières: les lieux du sida ", L'Esprit Créateur, vol. XXXVII, n 3, automne, 83-93.

Dreuilhe, A. E. [1987] : Corps à corps. Journal de sida, Paris, Gallimard.
Ducrot, O. [1984] : «Esquisse d'une théorie polyphonique de l'énonciation", dans Le Dire et le Dit, Paris, Éd. de Minuit, 171-233. GARCIN, J. [1990]: «La littérature a-t-elle tous les droits?",

L'Événement du Jeudi, 278, $1^{\text {er }}$ au 7 mars, 80-81.

Genette, G. [1982] : Palimpsestes. La littérature au second degré, Paris, Éd. du Seuil.

Guibert, H. [1990]: À l'ami qui ne m'a pas sauvé la vie, Paris, Gallimard.

HaWkINS, P. S. [1993]: "Naming Names: the Art of Memory and the NAmES Project AIDS Quilt», Critical Inquiry, vol. 19, nº 4, été, 752-779. KAPFERER, J.-N. [ 1987] : Rumeurs. Le plus vieux média du monde, Paris, Éd. du Seuil.

LEJEUne, P. [1986] : "L'image de l'auteur dans les médias ", dans Moi aussi, Paris, Éd. du Seuil, 87-99.

Merlin, H. [1994] : Public et littérature au XVIIe siècle, Paris, Les Belles Lettres.

RIEUSSET-LeMARIÉ, I. [1992]: Une fin de siècle épidémique, Arles, Actes Sud.

SAMSON, B. [1994]: On est pas sérieux quand on a dix-sept ans, Paris, Fixot.

STRAUSS, F. [1993]: "Un art neuf», Cahiers du cinéma, n 466, avril, 5-7. THÉRIEN, G. [1989]: "Discours ethnologique et discours fictionnel ", TLE, n6, 139-157. 